\title{
CONDITIONAL TRANSFORM WITH RESPECT TO THE GAUSSIAN PROCESS INVOLVING THE CONDITIONAL CONVOLUTION PRODUCT AND THE FIRST VARIATION
}

\author{
Hyun Soo Chung, Il Yong Lee, and Seung Jun Chang
}

\begin{abstract}
In this paper, we define a conditional transform with respect to the Gaussian process, the conditional convolution product and the first variation of functionals via the Gaussian process. We then examine various relationships of the conditional transform with respect to the Gaussian process, the conditional convolution product and the first variation for functionals $F$ in $S_{\alpha}[5,8]$.
\end{abstract}

\section{Introduction}

Let $C_{0}[0, T]$ denote one-parameter Wiener space; that is the space of realvalued continuous functions $x(t)$ on $[0, T]$ with $x(0)=0$. Let $\mathcal{M}$ denote the class of all Wiener measurable subsets of $C_{0}[0, T]$, and let $m$ denote Wiener measure. $\left(C_{0}[0, T], \mathcal{M}, m\right)$ is a complete measure space, and we denote the Wiener integral of a Wiener integrable functional $F$ by

$$
\int_{C_{0}[0, T]} F(x) d m(x) \text {. }
$$

A subset $\mathcal{B}$ of $C_{0}[0, T]$ is said to be scale-invariant measurable provided $\rho \mathcal{B}$ is $\mathcal{M}$-measurable for all $\rho>0$, and a scale-invariant measurable set $\mathcal{N}$ is said to be a scale-invariant null set provided $m(\rho \mathcal{N})=0$ for all $\rho>0$. A property that holds except on a scale-invariant null set is said to hold scale-invariant almost everywhere (s-a.e.) [10].

In $[5,8]$, the authors introduced the concept of Banach algebra $S_{\alpha}$. In [8], the authors studied the generalized integral transform (GIT) and the generalized convolution product (GCP) of functionals in $S_{\alpha}$. Additionally, they established some properties for the GIT and GCP. In [5], the authors obtained the conditional integral transform and the conditional convolution product of

Received April 16, 2013; Revised May 16, 2014.

2010 Mathematics Subject Classification. Primary 60J25, 28C20.

Key words and phrases. Brownian motion process, Wiener integral, Gaussian process, conditional convolution product, simple formula, conditional transform with respect to Gaussian process.

The present research was conducted by the research fund of Dankook University in 2014. 
functionals in $S_{\alpha}$. They established relationships between two of the three concepts of conditional integral transform, the conditional convolution product and the first variation. Recently, in [14], the authors introduced the transform with respect to the Gaussian process and the conditional convolution product.

In this paper, we introduce a conditional transform with respect to the Gaussian process and conditional convolution product. We then establish relationships between the conditional transform with respect to the Gaussian process of the conditional convolution product and the first variation. In Section 3, we establish the conditional transform with respect to the Gaussian process involving the functional of a functional in $S_{\alpha}$. We also obtain the conditional convolution product of functionals in $S_{\alpha}$. In Section 4, we establish the relationships between two of the three concepts of the conditional transform, the conditional convolution product, and first variation of the functionals. In Section 5, we establish all relationships between all three of these concepts.

\section{Definitions and preliminaries}

In this section, we list some definitions and properties from $[5,8,11,12,14]$.

Now we state definitions and notations which are needed to understand this paper.

(1) For $h \in L^{2}[0, T]$, we define the Gaussian process $Z_{h}$ by

$$
Z_{h}(x, t)=\int_{0}^{t} h(s) \tilde{d} x(t),
$$

where $\int_{0}^{t} h(s) \tilde{d} x(t)$ denotes the PWZ integral. For each $v \in L^{2}[0, T]$, let $\langle v, x\rangle=$ $\int_{0}^{T} v(t) \tilde{d} x(t)$. From [6], we note that

$$
\left\langle v, Z_{h}(x, \cdot)\right\rangle=\langle v h, x\rangle
$$

for $h \in L_{\infty}[0, T]$ and s-a.e. $x \in C_{0}[0, T]$. Thus, throughout this paper, we require $h$ to be in $L_{\infty}[0, T]$ rather than simply in $L^{2}[0, T]$.

(2) For all $v \in L^{2}[0, T]$, let

$$
B_{v}=\frac{1}{T} \int_{0}^{T} v(t) d t .
$$

(3) Let $K_{0}[0, T]$ be the set of all complex-valued continuous functions $x(t)$ defined on $[0, T]$ which vanish at $t=0$ and whose real and imaginary parts are elements of $C_{0}[0, T]$; namely,

$K_{0}[0, T]=\left\{x:[0, T] \rightarrow \mathbb{C} \mid x(0)=0, \operatorname{Re}(x) \in C_{0}[0, T]\right.$ and $\left.\operatorname{Im}(x) \in C_{0}[0, T]\right\}$.

Thus $C_{0}[0, T]$ is the subspace of all real-valued functions in $K_{0}[0, T]$.

(4) Let $\mathbb{C}$ be the class of all complex numbers. For each $\alpha \in \mathbb{C}$, let

$$
E_{\alpha} \equiv\left\{(\gamma, \beta) \in \mathbb{C} \times \mathbb{C}: \operatorname{Re}\left(\alpha^{2} \gamma^{2}\right) \leq 0 \text { and } \operatorname{Re}\left(\alpha^{2} \beta^{2}\right) \leq 0\right\}
$$

Now, we state the definitions of the transform with respect to the Gaussian process, the conditional convolution product and the first variation. 
Definition 2.1. Let $F$ and $G$ be functionals on $K_{0}[0, T]$ and let $\gamma, \beta, \rho$ and $\tau$ be non-zero complex numbers. Then the transform with respect to the Gaussian process, the convolution product and the first variation are defined by formulas

$$
\begin{gathered}
\left(T_{\gamma, \beta}^{h_{1}, h_{2}}(F)\right)(y)=\int_{C_{0}[0, T]} F\left(\gamma Z_{h_{1}}(x, \cdot)+\beta Z_{h_{2}}(y, \cdot)\right) d m(x), \\
\left(\left(F *_{s_{1} s_{2}} G\right)_{\rho, \tau}\right)(y)=\int_{C_{0}[0, T]} F\left(\tau Z_{s_{2}}(y, \cdot)+\rho Z_{s_{1}}(x, \cdot)\right) \\
\cdot G\left(\tau Z_{s_{2}}(y, \cdot)-\rho Z_{s_{1}}(x, \cdot)\right) d m(x), \\
\delta F\left(Z_{h}(x, \cdot) \mid Z_{s}(z, \cdot)\right)=\left.\frac{\partial}{\partial k} F\left(Z_{h}(x, \cdot)+k Z_{s}(z, \cdot)\right)\right|_{k=0}
\end{gathered}
$$

if they exist.

Remark 2.2. (1) When $h_{1}(t)=h_{2}(t)=1$ on $[0, T], T_{\gamma, \beta}^{1,1}$ is the integral transform used by Kim and Skoug [13]. In particular, $T_{1, i}^{1,1}(F)$ is the Fourier-Wiener transform introduced by Cameron in [1]. Also, $T_{\sqrt{2}, i}^{1,1}(F)$ is the Fourier-Wiener transform used by Cameron and Martin [2].

(2) If $s_{1}(t)=s_{2}(t)=1$ on $[0, T], \tau=\frac{1}{\sqrt{2}}$ and $\rho=\frac{1}{\sqrt{2 \lambda}}$ for $\lambda \in \tilde{\mathbb{C}}_{+}=\{\lambda \in$ $\mathbb{C}: \lambda \neq 0$ and $\operatorname{Re}(\lambda) \geq 0\}$, then the convolution product $\left(F *{ }_{11} G\right)_{\rho, \tau}$ coincides with convolution product $(F * G)_{\lambda}[3,4,7,8]$; that is to say, $\left(F *_{11} G\right)_{\rho, \tau}=$ $(F * G)_{\lambda}$ for $\lambda \in \tilde{\mathbb{C}}_{+}$.

(3) If $h(t)=s(t)=1$ on $[0, T]$, then the first variation of $F$ with respect to the Gaussian process coincides with the first variation of $F[5]$.

Let $X$ be a $\mathbb{R}$-valued function on $C_{0}[0, T]$ whose probability distribution $m_{X}$ is absolutely continuous with respect to Lebesgue measure on $\mathbb{R}$. Let $F$ be a $\mathbb{C}$-valued $m$-integrable functional on $C_{0}[0, T]$. Then the conditional integral of $F$ given $X$, denoted by $E[F \| X](\eta)$, is a Lebesgue measurable function of $\eta$, unique up to null sets in $\mathbb{R}$, satisfying the equation

$$
\int_{X^{-1}(B)} F(x) d m(x)=\int_{B} E[F \| X](\eta) d m_{X}(\eta)
$$

for all Borel sets $B$ in $\mathbb{R}$.

Throughout this paper, we will condition by the function $X: C_{0}[0, T] \rightarrow \mathbb{R}$ given by

$$
X(x)=x(T) .
$$

In [15], Park and Skoug gave a simple formula for expression conditional function space integrals in terms of an ordinary function space integrals by the formula

$$
E[F \| X](\eta)=\int_{C_{0}[0, T]} F\left(x(\cdot)-\frac{\dot{T}}{T} x(T)+\frac{\dot{T}}{T} \eta\right) d m(x)
$$


The following Wiener integral is used several times in this paper. For each $\alpha \in \mathbb{C}$ and for $v \in L^{2}[0, T]$

$$
\int_{C_{0}[0, T]} \exp \{\alpha\langle v, x\rangle\} d m(x)=\exp \left\{\frac{\alpha^{2}}{2}\|v\|_{2}^{2}\right\} .
$$

Next, we give the definition of the conditional transform with respect to the Gaussian process.

For each partition $\tau=\left\{t_{1}, \ldots, t_{n}\right\}$ of $[0, T]$ with $0=t_{0}<t_{1}<\cdots<t_{n}=T$, let $X_{\tau}: C_{0}[0, T] \rightarrow \mathbb{R}^{n}$ be defined by

$$
X_{\tau}(x)=\left(x\left(t_{1}\right), \ldots, x\left(t_{n}\right)\right) .
$$

Define a function $[x]$ on $[0, T]$ by

$$
[x](t)=x\left(t_{j-1}\right)+\frac{t-t_{j-1}}{t_{j}-t_{j-1}}\left(x\left(t_{j}\right)-x\left(t_{j-1}\right)\right)
$$

for $t_{j-1} \leq t \leq t_{j}$. Similarly, for $\vec{\eta}=\left(\eta_{1}, \ldots, \eta_{n}\right) \in \mathbb{R}^{n+1}$, define the function $[\vec{\eta}]$ of $\vec{\eta}$ on $[0, T]$ by

$$
[\vec{\eta}](t)=\eta_{j-1}+\frac{t-t_{j-1}}{t_{j}-t_{j-1}}\left(\eta_{j}-\eta_{j-1}\right),
$$

where $t_{j-1} \leq t \leq t_{j}$. Then both $[x]$ and $[\vec{\eta}]$ are continuous on $[0, T]$, they are line segments on each interval $\left[t_{j-1}, t_{j}\right]$, and $[x]\left(t_{j}\right)=x\left(t_{j}\right)$ and $[\vec{\eta}]\left(t_{j}\right)=\eta_{j}$ at each $t_{j}$.

Definition 2.3. Let $F$ be a functional defined on $K_{0}[0, T]$ and let $X$ be given by equation (2.8). For each non-zero complex numbers $\gamma$ and $\beta$, the conditional transform with respect to the Gaussian process $T_{\gamma, \beta}^{h_{1}, h_{2}}(F \| X)(y, \eta)$ of $F$ given $X$ is given by the formula

$$
T_{\gamma, \beta}^{h_{1}, h_{2}}(F \| X)(y, \eta)=\int_{C_{0}[0, T]} F\left(\gamma Z_{h_{1}}(x-[x]+[\vec{\eta}], \cdot)+\beta Z_{h_{2}}(y, \cdot)\right) d m(x)
$$

for $y \in K_{0}[0, T]$ and $\eta \in \mathbb{R}$, if it exists. In particular, if $X$ is given by equation (2.6), then

$$
\begin{aligned}
& T_{\gamma, \beta}^{h_{1}, h_{2}}(F \| X)(y, \eta) \\
= & \int_{C_{0}[0, T]} F\left(\gamma Z_{h_{1}}\left(x(\cdot)-\frac{\dot{T}}{T} x(T)+\frac{\dot{T}}{T} \eta, \cdot\right)+\beta Z_{h_{2}}(y, \cdot)\right) d m(x) .
\end{aligned}
$$

The following simple example illustrates Definition 2.3 above.

Example 2.4. Define the functional $F: K_{0}[0, T] \rightarrow \mathbb{C}$ by $F(x)=\exp \{\langle v, x\rangle\}$ for $v \in L^{2}[0, T]$. Then

$$
\begin{aligned}
& T_{\gamma, \beta}^{h_{1}, h_{2}}(F \| X)(y, \eta) \\
= & \int_{C_{0}[0, T]} F\left(\gamma Z_{h_{1}}\left(x(\cdot)-\frac{\cdot}{T} x(T)+\frac{\dot{T}}{T} \eta, \cdot\right)+\beta Z_{h_{2}}(y, \cdot)\right) d m(x)
\end{aligned}
$$




$$
\begin{aligned}
& =\int_{C_{0}[0, T]} \exp \left\{\gamma\left\langle v, Z_{h_{1}}\left(x(\cdot)-\frac{\dot{T}}{T} x(T)+\frac{\dot{T}}{T} \eta, \cdot\right)\right\rangle+\beta\left\langle v h_{2}, y\right\rangle\right\} d m(x) \\
& =\exp \left\{\beta\left\langle v h_{2}, y\right\rangle+\gamma \eta B_{v h_{1}}\right\} \int_{C_{0}[0, T]} \exp \left\{\gamma\left\langle v h_{1}-B_{v h_{1}}, x\right\rangle\right\} d m(x) \\
& =\exp \left\{\beta\left\langle v h_{2}, y\right\rangle+\frac{\gamma^{2}}{2}\left\|v h_{1}-B_{v h_{1}}\right\|_{2}^{2}+\gamma \eta B_{v h_{1}}\right\} .
\end{aligned}
$$

Next, we state the definition of the conditional convolution product of functionals $F$ and $G$ on $K_{0}[0, T]$.

Definition 2.5. Let $F$ and $G$ be functionals defined on $K_{0}[0, T]$ and let $X$ be given by equation (2.6). For each non-zero complex numbers $\rho$ and $\tau$, the conditional convolution product $\left(\left(F *_{s_{1} s_{2}} G\right)_{\rho, \tau} \| X\right)(y, \eta)$ of $F$ and $G$ given $X$ is given by the formula

$$
\begin{aligned}
& \left(\left(F *_{s_{1} s_{2}} G\right)_{\rho, \tau} \| X\right)(y, \eta) \\
= & \int_{C_{0}[0, T]} F\left(\tau Z_{s_{2}}(y, \cdot)+\rho Z_{s_{1}}\left(x(\cdot)-\frac{\cdot}{T} x(T)+\frac{\dot{ }}{T} \eta, \cdot\right)\right) \\
& \cdot G\left(\tau Z_{s_{2}}(y, \cdot)-\rho Z_{s_{1}}\left(x(\cdot)-\frac{\dot{T}}{T} x(T)+\frac{\dot{T}}{T} \eta, \cdot\right)\right) d m(x)
\end{aligned}
$$

for $y \in K_{0}[0, T]$ and $\eta \in \mathbb{R}$, if it exists.

Remark 2.6. (1) When $h_{1}(t)=h_{2}(t)=1$ on $[0, T], T_{\gamma, \beta}^{1,1}(F \| X)(y, \eta)$ is the conditional integral transform $\mathcal{F}_{\gamma, \beta}(F \| X)(y, \eta)$ introduced by Chung, Choi and Chang [5].

(2) When $s_{1}(t)=s_{2}(t)=1$ on $[0, T], \tau=\frac{1}{\sqrt{2}}$ and $\rho=\frac{\gamma}{\sqrt{2}},\left(\left(F *_{11}\right.\right.$ $\left.G)_{\rho, \tau} \| X\right)(y, \eta)$ is the conditional convolution product $\left((F * G)_{\gamma} \| X\right)(y, \eta)$ introduced by Chung, Choi and Chang [5].

\section{Conditional transform with respect to the Gaussian process on function space}

In this section, we establish the conditional transform with respect to the Gaussian process for the functional $F$ in $S_{\alpha}$. We then obtain the conditional transform with respect to the Gaussian process involving the conditional convolution product and the first variation.

Let $\mathcal{M}\left(L^{2}[0, T]\right)$ be the space of $\mathbb{C}$-valued, countably additive (and hence finite) Borel measures on $L^{2}[0, T]\left[9\right.$, pp. 126-127] and [16, p. 119]. $\mathcal{M}\left(L^{2}[0, T]\right)$ is a Banach algebra under the total variation norm and with convolution as multiplication. For each complex number $\alpha$ with $\operatorname{Re}\left(\alpha^{2}\right) \leq 0$, let $S_{\alpha}$ be the class of functionals of the form

$$
F(x)=\int_{L^{2}[0, T]} \exp \{\alpha\langle v, x\rangle\} d f(v)
$$

for s-a.e. $x \in C_{0}[0, T]$, where $f \in \mathcal{M}\left(L^{2}[0, T]\right)[5,8]$. 
Remark 3.1. (1) For each complex number $\alpha$ with $\operatorname{Re}\left(\alpha^{2}\right) \leq 0$, using formula (2.7) above, we have

$$
\int_{C_{0}[0, T]} \int_{L^{2}[0, T]} \exp \{\alpha\langle v, x\rangle\} d f(v) d m(x)=\int_{L^{2}[0, T]} \exp \left\{\frac{\alpha^{2}}{2}\|v\|_{2}^{2}\right\} d f(v)<\infty
$$

since $\left|\exp \left\{\frac{\alpha^{2}}{2}\|v\|_{2}^{2}\right\}\right| \leq 1$. This tells us that $\int_{L^{2}[0, T]} \exp \{\alpha\langle v, x\rangle\} d f(v)$ exists for a.e. $x \in C_{0}[0, T]$. Furthermore, for all real number $\rho>0$,

$\int_{C_{0}[0, T]} \int_{L^{2}[0, T]} \exp \{\alpha\langle v, \rho x\rangle\} d f(v) d m(x)=\int_{L^{2}[0, T]} \exp \left\{\frac{\rho^{2} \alpha^{2}}{2}\|v\|_{2}^{2}\right\} d f(v)<\infty$ because $\operatorname{Re}\left(\rho^{2} \alpha^{2}\right) \leq 0$. Hence the functional $F$ given by equation (3.1) is well defined for s-a.e. $x \in C_{0}[0, T]$.

(2) The map $f \rightarrow F$ defined by (3.1) sets up on algebra isomorphism between $\mathcal{M}\left(L^{2}[0, T]\right)$ and $S_{\alpha}$. In this case, $S_{\alpha}$ becomes a Banach algebra under the norm $\|F\|=\|f\|$.

(3) Note that for $F \in S_{\alpha}$, the function $G: C_{0}[0, T] \rightarrow \mathbb{C}$ given by $G(x)=$ $F\left(Z_{h}(x, \cdot)\right)$ with $h \in L_{\infty}[0, T]$, belongs to the Banach algebra $S_{\alpha}$, see [6].

Remark 3.2 ([5, Remark 2.3]). (1) First we could consider the following integral

$$
\int_{L^{2}[0, T]} \exp \left\{\alpha\langle v, x\rangle+\xi B_{v}\right\} d f(v), \quad \xi \in \mathbb{C} .
$$

If we assume that

$$
\int_{L^{2}[0, T]} \exp \left\{|\xi| \int_{0}^{T}|v(t)| d t\right\}|d f(v)|<\infty
$$

for all complex number $\xi$, then

$$
\int_{L^{2}[0, T]} \exp \left\{\xi B_{v}\right\} d f(v)<\infty \text { and } \int_{L^{2}[0, T]} \exp \{\alpha\langle v, x\rangle\} d f(v)
$$

exists for s-a.e. $x \in C_{0}[0, T]$. However, the integral (3.2) might not exist because the product of $L^{1}[0, T]$-functionals might not be in $L^{1}[0, T]$.

(2) In this paper, we need a condition for $f$ in $\mathcal{M}\left(L^{2}[0, T]\right)$ to show the existence of the integral in equation (3.2).

(i) If $v \in L^{2}[0, T]$ is a function of bounded variation, then for each $x \in$ $C_{0}[0, T]$

$$
|\langle v, x\rangle| \leq\|x\|_{\infty}\left(|v(T)|+V_{0}^{T}(v)\right)<\infty,
$$

where $V_{0}^{T}(v)$ is the total variation of $v$ on $[0, T]$. Hence if we assume that

$$
\int_{L^{2}[0, T]} \exp \left\{|\xi|\left[|v(T)|+\left|V_{0}^{T}(v)\right|+\int_{0}^{T}|v(t)| d t\right]\right\}|d f(v)|<\infty,
$$

then the integral (3.2) always exists.

(ii) Let $v$ be an element of $L^{2}[0, T]$. Then we note that

$$
|\langle v, x\rangle|=\lim _{n \rightarrow \infty}\left|\left\langle v_{n}, x\right\rangle\right| \leq \lim _{n \rightarrow \infty}\|x\|_{\infty}\left(\left|v_{n}(T)\right|+V_{0}^{T}\left(v_{n}\right)\right),
$$


where $v_{n}(t)=\sum_{k=1}^{n}\left(v, \phi_{k}\right)_{2} \phi_{k}(t),\left\{\phi_{k}\right\}$ is a complete orthonormal set in $L^{2}[0, T]$ and $(\cdot, \cdot)_{2}$ is the inner product on $L^{2}[0, T]$. Hence if we add a condition

$$
\lim _{n \rightarrow \infty}\left(\left|v_{n}(T)\right|+V_{0}^{T}\left(v_{n}\right)\right)
$$

exists, then we can obtain the existence of the integral (3.2) under the condition which is similar to the condition (3.4).

(3) As mentioned above, we can give the condition (3.6) because the expression (3.5) is independent of the choice of the complete orthonormal set $\left\{\phi_{k}\right\}$ and the all expressions in equation (3.5) exists for s-a.e. $x \in C_{0}[0, T]$. Hence, we assume that for $f \in \mathcal{M}\left(L^{2}[0, T]\right)$ which satisfies the condition (3.3) above, the integral (3.2) always exists.

In our next theorem, we obtain the conditional transform with respect to the Gaussian process of a functional in $S_{\alpha}$.

Theorem 3.3. Let $F$ be an element of $S_{\alpha}$. Let $X$ be given by equation (2.6). Then for all $(\gamma, \beta) \in E_{\alpha}$, the conditional transform with respect to the Gaussian process $T_{\gamma, \beta}^{h_{1}, h_{2}}(F \| X)$ exists and is given by the formula

$$
\begin{aligned}
& T_{\gamma, \beta}^{h_{1}, h_{2}}(F \| X)(y, \eta) \\
= & \int_{L^{2}[0, T]} \exp \left\{\alpha \beta\left\langle v h_{2}, y\right\rangle+\frac{\gamma^{2} \alpha^{2}}{2}\left\|v h_{1}-B_{v h_{1}}\right\|_{2}^{2}+\gamma \alpha B_{v h_{1}} \eta\right\} d f(v)
\end{aligned}
$$

for s-a.e. $y \in C_{0}[0, T]$ and a.e. $\eta \in \mathbb{R}$, where $B_{v h_{1}}$ is given by equation (2.2). Furthermore $T_{\gamma, \beta}^{h_{1}, h_{2}}(F \| X)$ is an element of $S_{\alpha \beta}$ with associated measure $\phi_{1}^{\eta}$ defined by

$$
\phi_{1}^{\eta}(E)=\int_{E} \exp \left\{\frac{\gamma^{2} \alpha^{2}}{2}\left\|v h_{1}-B_{v h_{1}}\right\|_{2}^{2}+\gamma \alpha B_{v h_{1}} \eta\right\} d f(v)
$$

for $E \in \mathcal{B}\left(L^{2}[0, T]\right)$.

Proof. Using equations (2.9) and (3.1), we have

$$
\begin{aligned}
& T_{\gamma, \beta}^{h_{1}, h_{2}}(F \| X)(y, \eta) \\
= & \int_{C_{0}[0, T]} \int_{L^{2}[0, T]} \exp \left\{\alpha \gamma\left\langle v h_{1}-B_{v h_{1}}, x\right\rangle+\alpha \gamma \eta B_{v h_{1}}+\alpha \beta\left\langle v h_{2}, y\right\rangle\right\} d f(v) d m(x) .
\end{aligned}
$$

Applying the Fubini theorem and equation (2.7) to the equation above, it follows that

$$
\begin{aligned}
& T_{\gamma, \beta}^{h_{1}, h_{2}}(F \| X)(y, \eta) \\
= & \int_{L^{2}[0, T]} \exp \left\{\alpha \beta\left\langle v h_{2}, y\right\rangle+\frac{\gamma^{2} \alpha^{2}}{2}\left\|v h_{1}-B_{v h_{1}}\right\|_{2}^{2}+\gamma \alpha B_{v h_{1}} \eta\right\} d f(v) .
\end{aligned}
$$

Since $(\gamma, \beta) \in E_{\alpha}$ and hypothesis of Remark 3.2, the last expression above exists. Thus the equation (3.7) is established. Also, the last expression above 
becomes

$$
T_{\gamma, \beta}^{h_{1}, h_{2}}(F \| X)(y, \eta)=\int_{L^{2}[0, T]} \exp \left\{\alpha \beta\left\langle v h_{2}, y\right\rangle\right\} d \phi_{1}^{\eta}(v) .
$$

Hence $T_{\gamma, \beta}^{h_{1}, h_{2}}(F \| X)$ is an element of $S_{\alpha \beta}$ because $\phi_{1}^{n}$ is an element of $\mathcal{M}\left(L^{2}[0, T]\right)$.

Remark 3.4. The main result in [5, Theorem 3.1] follows immediately from Theorem 3.3 above by choosing $h_{1}(t)=h_{2}(t)=1$ on $[0, T]$.

In our next theorem, we establish the double conditional transform involving the functional of a functional in $S_{\alpha}$.

Theorem 3.5. Let $F$ and $X$ be as in Theorem 3.3. Assume that $h_{3}(t)=$ $h_{1}(t) h_{4}(t)$ on $[0, T]$ and $\gamma_{2}=\gamma_{1} \beta_{2}$. Then for all $\left(\gamma_{2}, \beta_{2}\right) \in E_{\alpha},\left(\gamma_{1}, \beta_{1}\right) \in E_{\alpha \beta_{2}}$ and $\left(\gamma_{2}, \beta_{1} \beta_{2}\right) \in E_{\alpha}$,

$$
T_{\gamma_{1}, \beta_{1}}^{h_{1}, h_{2}}\left(T_{\gamma_{2}, \beta_{2}}^{h_{3}, h_{4}}(F \| X)\left(\cdot, \eta_{1}\right) \| X\right)\left(y, \eta_{2}\right)=T_{\sqrt{2} \gamma_{2}, \beta_{1} \beta_{2}}^{h_{3}, h_{2} h_{4}}(F \| X)\left(y, \frac{\eta_{2}+\eta_{1}}{\sqrt{2}}\right)
$$

for s-a.e. $y \in C_{0}[0, T]$ and a.e. $\eta_{1}, \eta_{2} \in \mathbb{R}$. Also, both sides of the expressions in equation (3.9) are given by the formula

$$
\int_{L^{2}[0, T]} \exp \left\{\alpha \beta_{1} \beta_{2}\left\langle v h_{2} h_{4}, y\right\rangle+\alpha^{2} \gamma_{2}^{2}\left\|v h_{3}-B_{v h_{3}}\right\|_{2}^{2}+\alpha \gamma_{2}\left(\eta_{2}+\eta_{1}\right) B_{v h_{3}}\right\} d f(v) \text {. }
$$

Furthermore, $T_{\sqrt{2} \gamma_{2}, \beta_{1} \beta_{2}}^{h_{3}, h_{2} h_{4}}(F \| X)$ is an element of $S_{\alpha \beta_{1} \beta_{2}}$ with associated measure $\phi_{2}^{\eta}$ defined by

$$
\phi_{2}^{\eta}(E)=\int_{E} \exp \left\{\alpha^{2} \gamma_{2}^{2}\left\|v h_{3}-B_{v h_{3}}\right\|_{2}^{2}+\alpha \gamma_{2}\left(\eta_{2}+\eta_{1}\right) B_{v h_{3}}\right\} d f(v)
$$

for $E \in \mathcal{B}\left(L^{2}[0, T]\right)$.

Proof. By using equations (2.9) and (3.1), we have

$$
\begin{aligned}
& T_{\gamma_{1}, \beta_{1}}^{h_{1}, h_{2}}\left(T_{\gamma_{2}, \beta_{2}}^{h_{3}, h_{4}}(F \| X)\left(\cdot, \eta_{1}\right) \| X\right)\left(y, \eta_{2}\right) \\
& =\int_{C_{0}[0, T]} \int_{C_{0}[0, T]}\left[\int _ { L ^ { 2 } [ 0 , T ] } \operatorname { e x p } \left\{\alpha \beta_{1} \beta_{2}\left\langle v h_{2} h_{4}, y\right\rangle+\alpha \gamma_{2}\left\langle v h_{3}-B_{v h_{3}}, w\right\rangle\right.\right. \\
& +\alpha \gamma_{2} \eta_{1} B_{v h_{3}}+\alpha \gamma_{1} \beta_{2}\left\langle v h_{1} h_{4}-B_{v h_{1} h_{4}}, x\right\rangle \\
& \left.\left.+\alpha \gamma_{1} \beta_{2} \eta_{2} B_{v h_{1} h_{4}}\right\} d f(v)\right] d m(x) d m(w) \\
& =\int_{L^{2}[0, T]} \exp \left\{\alpha \beta_{1} \beta_{2}\left\langle v h_{2} h_{4}, y\right\rangle+\alpha \gamma_{2} \eta_{1} B_{v h_{3}}+\alpha \gamma_{1} \beta_{2} \eta_{2} B_{v h_{1} h_{4}}\right\} \\
& \text {. }\left[\int_{C_{0}[0, T]} \exp \left\{\alpha \gamma_{1} \beta_{2}\left\langle v h_{1} h_{4}-B_{v h_{1} h_{4}}, x\right\rangle\right\} d m(x)\right] \\
& \cdot\left[\int_{C_{0}[0, T]} \exp \left\{\alpha \gamma_{2}\left\langle v h_{3}-B_{v h_{3}}, w\right\rangle\right\} d m(w)\right] d f(v)
\end{aligned}
$$


CONDITIONAL TRANSFORM WITH RESPECT TO THE GAUSSIAN PROCESS 1569

$$
\begin{aligned}
= & \int_{L^{2}[0, T]} \exp \left\{\alpha \beta_{1} \beta_{2}\left\langle v h_{2} h_{4}, y\right\rangle+\alpha \gamma_{2} \eta_{1} B_{v h_{3}}+\alpha \gamma_{1} \beta_{2} \eta_{2} B_{v h_{1} h_{4}}\right. \\
& \left.+\frac{\alpha^{2} \gamma_{1}^{2} \beta_{2}^{2}}{2}\left\|v h_{1} h_{4}-B_{v h_{1} h_{4}}\right\|_{2}^{2}+\frac{\alpha^{2} \gamma_{2}^{2}}{2}\left\|v h_{3}-B_{v h_{3}}\right\|_{2}^{2}\right\} .
\end{aligned}
$$

Since $h_{3}(t)=h_{1}(t) h_{4}(t)$ on $[0, T]$ and $\gamma_{2}=\gamma_{1} \beta_{2}$, the last expression equation above is equal to

$$
\int_{L^{2}[0, T]} \exp \left\{\alpha \beta_{1} \beta_{2}\left\langle v h_{2} h_{4}, y\right\rangle+\alpha^{2} \gamma_{2}^{2}\left\|v h_{3}-B_{v h_{3}}\right\|_{2}^{2}+\alpha \gamma_{2}\left(\eta_{2}+\eta_{1}\right) B_{v h_{3}}\right\} d f(v) .
$$

Hence by Definition 2.3, we have

$$
T_{\gamma_{1}, \beta_{1}}^{h_{1}, h_{2}}\left(T_{\gamma_{2}, \beta_{2}}^{h_{3}, h_{4}}(F \| X)\left(\cdot, \eta_{1}\right) \| X\right)\left(y, \eta_{2}\right)=T_{\sqrt{2} \gamma_{2}, \beta_{1} \beta_{2}}^{h_{3}, h_{2} h_{4}}(F \| X)\left(y, \frac{\eta_{2}+\eta_{1}}{\sqrt{2}}\right) .
$$

Thus the equation (3.9) is established. Using the similar method in the proof of Theorem 3.3, both sides of the equation (3.5) exists. Also, equation (3.10) becomes

$$
\int_{L^{2}[0, T]} \exp \left\{\alpha \beta_{1} \beta_{2}\left\langle v h_{2} h_{4}, y\right\rangle\right\} d \phi_{2}^{\eta}(v) .
$$

Hence equation (3.9) above is an element of $S_{\alpha \beta_{1} \beta_{2}}$ because $\phi_{2}^{\eta}$ is an element of $\mathcal{M}\left(L^{2}[0, T]\right)$.

In our next theorem, we obtain the conditional convolution product of functionals in $S_{\alpha}$.

Theorem 3.6. Let $F$ and $G$ be elements of $S_{\alpha}$. Let $X$ be given by equation (2.6). Then for all $(\rho, \tau) \in E_{\alpha}$, the conditional convolution product $\left(\left(F *_{s_{1} s_{2}}\right.\right.$ $\left.G)_{\rho, \tau} \| X\right)$ exists and is given by the formula

$$
\begin{gathered}
=\int_{L^{2}[0, T]} \int_{L^{2}[0, T]} \exp \left\{\alpha \tau \left\langle\left\langle(v+u) s_{2}, y\right\rangle+\frac{\alpha^{2} \rho^{2}}{2}\left\|(v-u) s_{1}-B_{(v-u) s_{1}}\right\|_{2}^{2}\right.\right. \\
\left.+\alpha \rho \eta B_{(v-u) s_{1}}\right\} d f(v) d g(u)
\end{gathered}
$$

for s-a.e. $y \in C_{0}[0, T]$ and a.e. $\eta \in \mathbb{R}$. Furthermore $\left(\left(F *_{s_{1} s_{2}} G\right)_{\rho, \tau} \| X\right)$ is an element of $S_{\alpha \tau}$.

Proof. By using equations (2.7) and (2.10), we have

$$
\begin{aligned}
& \left(\left(F *_{s_{1} s_{2}} G\right)_{\rho, \tau} \| X\right)(y, \eta) \\
= & \int_{C_{0}[0, T]} F\left(\tau Z_{s_{2}}(y, \cdot)+\rho Z_{s_{1}}\left(x(\cdot)-\frac{\dot{T}}{T} x(T)+\frac{\dot{T}}{T} \eta\right)\right) \\
& \cdot G\left(\tau Z_{s_{2}}(y, \cdot)-\rho Z_{s_{1}}\left(x(\cdot)-\frac{\dot{T}}{T} x(T)+\frac{\cdot}{T} \eta\right)\right) d m(x) \\
= & \int_{L^{2}[0, T]} \int_{L^{2}[0, T]} \exp \left\{\alpha \tau\left\langle(v+u) s_{2}, y\right\rangle+\alpha \rho \eta\left(B_{v s_{1}}-B_{u s_{1}}\right)\right\}
\end{aligned}
$$




$$
\begin{aligned}
& \cdot\left[\int_{C_{0}[0, T]} \exp \left\{\alpha \rho\left\langle(v-u) s_{1}-\left(B_{v s_{1}}-B_{u s_{1}}\right), x\right\rangle\right\} d m(x)\right] d f(v) d g(u) \\
= & \int_{L^{2}[0, T]} \int_{L^{2}[0, T]} \exp \left\{\alpha \tau\left\langle(v+u) s_{2}, y\right\rangle+\alpha \rho \eta B_{(v-u) s_{1}}\right. \\
& \left.+\frac{\alpha^{2} \rho^{2}}{2}\left\|(v-u) s_{1}-B_{(v-u) s_{1}}\right\|_{2}^{2}\right\} d f(v) d g(u) .
\end{aligned}
$$

Let a set function $\phi_{3}^{\eta}: \mathcal{B}\left(L^{2}[0, T] \times L^{2}[0, T]\right) \rightarrow \mathbb{C}$ be defined by

$$
\phi_{3}^{\eta}(E)=\int_{E} \exp \left\{\frac{\alpha^{2} \rho^{2}}{2}\left\|(v-u) s_{1}-B_{(v-u) s_{1}}\right\|_{2}^{2}+\alpha \rho \eta B_{(v-u) s_{1}}\right\} d f(v) d g(u)
$$

for $E \in \mathcal{B}\left(L^{2}[0, T] \times L^{2}[0, T]\right)$. Then $\phi_{3}^{\eta}$ is a complex Borel measure on $\mathcal{B}\left(L^{2}[0, T] \times L^{2}[0, T]\right)$. Now we define a function $\varphi: L^{2}[0, T] \times L^{2}[0, T] \rightarrow$ $L^{2}[0, T]$ by $\varphi(u, v)=u+v$. Let $\tilde{\phi}=\phi_{3}^{\eta} \circ \varphi^{-1}$. Then $\tilde{\phi}$ belongs to $\mathcal{M}\left(L^{2}[0, T]\right)$ since $(\rho, \tau) \in E_{\alpha}$. So

$$
\left(\left(F *_{s_{1} s_{2}} G\right)_{\rho, \tau} \| X\right)(y, \eta)=\int_{L^{2}[0, T]} \exp \left\{\alpha \tau\left\langle w s_{2}, y\right\rangle\right\} d \tilde{\phi}(w) .
$$

Hence $\left(\left(F *_{s_{1} s_{2}} G\right)_{\rho, \tau} \| X\right)$ exists and is given by (3.11) and it belongs to $S_{\alpha \tau}$.

Remark 3.7. The main result in [5, Theorem 3.3] follows immediately from Theorem 3.6 above by choosing $s_{1}(t)=s_{2}(t)=1$ on $[0, T], \tau=\frac{1}{\sqrt{2}}$ and $\rho=\frac{\gamma}{\sqrt{2}}$.

\section{Relationships between two concepts}

In Section 3, we introduced the conditional transform with respect to the Gaussian process and the conditional convolution product. In this section, we establish the relationships between exactly two of three concepts of the conditional transform, conditional convolution product and the first variation for functionals.

In our next theorem, we obtain the conditional transform with respect to the Gaussian process involving the conditional convolution product of functionals in $S_{\alpha}$.

Theorem 4.1. Let $F, G$ and $X$ be as in Theorem 3.3. Assume that $\tau \gamma=\rho$ and $h_{1}(t) s_{2}(t)=s_{1}(t)$ on $[0, T]$. Then for all $(\rho, \tau) \in E_{\alpha},(\gamma, \beta) \in E_{\alpha \tau}$ and $(\rho, \beta \tau) \in E_{\alpha}$,

$$
\begin{aligned}
& T_{\gamma, \beta}^{h_{1}, h_{2}}\left(\left(\left(F *_{s_{1} s_{2}} G\right)_{\rho, \tau} \| X\right)\left(\cdot, \eta_{1}\right) \| X\right)\left(y, \eta_{2}\right) \\
= & T_{\sqrt{2} \rho, \beta \tau}^{s_{1}, h_{2} s_{2}}(F \| X)\left(y, \frac{\eta_{2}+\eta_{1}}{\sqrt{2}}\right) T_{\sqrt{2} \rho, \beta \tau}^{s_{1}, h_{2} s_{2}}(G \| X)\left(y, \frac{\eta_{2}-\eta_{1}}{\sqrt{2}}\right)
\end{aligned}
$$

for s-a.e. $y \in C_{0}[0, T]$ and a.e. $\eta_{1}, \eta_{2} \in \mathbb{R}$. Also, both sides of the expression in equation (4.1) are given by the formula

$$
\int_{L^{2}[0, T]} \int_{L^{2}[0, T]} \exp \left\{\alpha \beta \tau\left\langle(v+u) h_{2} s_{2}, y\right\rangle+\alpha^{2} \rho^{2}\left(\left\|v s_{1}-B_{v s_{1}}\right\|_{2}^{2}+\left\|u s_{1}-B_{u s_{1}}\right\|_{2}^{2}\right)\right.
$$


CONDITIONAL TRANSFORM WITH RESPECT TO THE GAUSSIAN PROCESS 1571

$$
\left.+\alpha \rho\left(\eta_{2} B_{(v+u) s_{1}}+\eta_{1} B_{(v-u) s_{1}}\right)\right\} d f(v) d g(u) .
$$

Proof. First by using equations (2.9), (2.10) and the Fubini theorem, we obtain that

$$
\begin{aligned}
& T_{\gamma, \beta}^{h_{1}, h_{2}}\left(\left(\left(F *_{s_{1} s_{2}} G\right)_{\rho, \tau} \| X\right)\left(\cdot, \eta_{1}\right) \| X\right)\left(y, \eta_{2}\right) \\
&= \int_{C_{0}[0, T]} \int_{C_{0}[0, T]} F\left(\tau \gamma Z_{h_{1} s_{2}}(x, \cdot)-\tau \gamma B_{h_{1} s_{2}} x(T)+\tau \gamma \eta_{2} B_{h_{1} s_{2}}\right. \\
&\left.\quad+\tau \beta Z_{h_{2} s_{2}}(y, \cdot)+\rho Z_{s_{1}}(w, \cdot)-\rho B_{s_{1}} w(T)+\rho \eta_{1} B_{s_{1}}\right) \\
& \cdot G\left(\tau \gamma Z_{h_{1} s_{2}}(x, \cdot)-\tau \gamma B_{h_{1} s_{2}} x(T)+\tau \gamma \eta_{2} B_{h_{1} s_{2}}\right. \\
&\left.\quad+\tau \beta Z_{h_{2} s_{2}}(y, \cdot)-\rho Z_{s_{1}}(w, \cdot)+\rho B_{s_{1}} w(T)-\rho \eta_{1} B_{s_{1}}\right) d m(w) d m(x) \\
&= \int_{L^{2}[0, T]} \int_{L^{2}[0, T]} \exp \left\{\alpha \tau \beta\left\langle(v+u) h_{2} s_{2}, y\right\rangle+\alpha \rho \eta_{1} B_{(v-u) s_{1}}+\alpha \tau \gamma \eta_{2} B_{(v+u) h_{1} s_{2}}\right\} \\
& \cdot\left[\int_{C_{0}[0, T]} \exp \left\{\alpha \tau \gamma\left\langle(v+u) h_{1} s_{2}-B_{(v+u) h_{1} s_{2}}, x\right\rangle\right\} d m(x)\right] \\
& \cdot\left[\int_{C_{0}[0, T]} \exp \left\{\alpha \rho \gamma\left\langle(v-u) s_{1}-B_{(v-u) s_{1}}, w\right\rangle\right\} d m(w)\right] d f(v) d g(u) \\
&= \int_{L^{2}[0, T]} \int_{L^{2}[0, T]} \exp \left\{\alpha \beta \tau\left\langle(v+u) h_{2} s_{2}, y\right\rangle+\alpha^{2} \rho^{2}\left(\left\|v s_{1}-B_{v s_{1}}\right\|_{2}^{2}\right.\right. \\
&\left.\left.\quad+\left\|u s_{1}-B_{u s_{1}}\right\|_{2}^{2}\right)+\alpha \rho\left(\eta_{2} B_{(v+u) s_{1}}+\eta_{1} B_{(v-u) s_{1}}\right)\right\} d f(v) d g(u) .
\end{aligned}
$$

Next, using equation (3.7), we have

$$
\begin{aligned}
& T_{\sqrt{2} \rho, \beta \tau}^{s_{1}, h_{2} s_{2}}(F \| X)\left(y, \frac{\eta_{2}+\eta_{1}}{\sqrt{2}}\right) \\
= & \int_{L^{2}[0, T]} \exp \left\{\alpha \beta \tau\left\langle v h_{2} s_{2}, y\right\rangle+\alpha^{2} \rho^{2}\left\|v s_{1}-B_{v s_{1}}\right\|_{2}^{2}+\alpha \rho\left(\eta_{2}+\eta_{1}\right) B_{v s_{1}}\right\} d f(v)
\end{aligned}
$$

and

$$
\begin{aligned}
& T_{\sqrt{2} \rho, \beta \tau}^{s_{1}, h_{2} s_{2}}(G \| X)\left(y, \frac{\eta_{2}-\eta_{1}}{\sqrt{2}}\right) \\
= & \int_{L^{2}[0, T]} \exp \left\{\alpha \beta \tau\left\langle u h_{2} s_{2}, y\right\rangle+\alpha^{2} \rho^{2}\left\|u s_{1}-B_{u s_{1}}\right\|_{2}^{2}+\alpha \rho\left(\eta_{2}-\eta_{1}\right) B_{u s_{1}}\right\} d g(u) .
\end{aligned}
$$

Thus, equation (4.1) follows from equations (4.2)-(4.4). Since $(\rho, \tau) \in E_{\alpha}$, $(\rho, \beta \tau) \in E_{\alpha}$ and hypothesis of Remark 3.2, the both sides of the expressions in equation (4.1) exists. 
Remark 4.2. The main result in [5, Theorem 3.3] follows immediately from Theorem 4.1 above by choosing $h_{j}(t)=s_{j}(t)=1(j=1,2)$ on $[0, T], \tau=\frac{1}{\sqrt{2}}$ and $\rho=\frac{\gamma}{\sqrt{2}}$.

The following theorem follows immediately from Theorems 3.5 and 4.1.

Theorem 4.3. Let $F$ and $X$ be as in Theorem 4.1. Assume that $\tau \gamma=\rho$, $\gamma=\sqrt{2} \rho \beta, s_{1}(t)=h_{1}(t) s_{2}(t)$ and $h_{1}(t)=s_{1}(t) h_{2}(t)$ on $[0, T]$. Then for all $(\gamma, \beta) \in E_{\alpha} \cap E_{\alpha \beta \tau},(\rho, \tau) \in E_{\alpha \beta}$, and $\left(\gamma, \beta^{2} \tau\right) \in E_{\alpha}$,

$$
\begin{aligned}
& T_{\gamma, \beta}^{h_{1}, h_{2}}\left(\left(\left(T_{\gamma, \beta}^{h_{1}, h_{2}}(F \| X)\left(\cdot, \eta_{1}\right) *_{s_{1} s_{2}} T_{\gamma, \beta}^{h_{1}, h_{2}}(G \| X)\left(\cdot, \eta_{2}\right)\right)_{\rho, \tau} \| X\right)\left(\cdot, \eta_{3}\right) \| X\right)\left(y, \eta_{4}\right) \\
= & T_{\sqrt{2} \gamma, \beta^{2} \tau}^{h_{1}, h_{2}^{2} s_{2}}(F \| X)\left(y, \frac{\eta_{4}+\eta_{3}+\sqrt{2} \eta_{1}}{2}\right) T_{\sqrt{2} \gamma, \beta^{2} \tau}^{h_{1}, h_{2}^{2} s_{2}}(G \| X)\left(y, \frac{\eta_{4}-\eta_{3}+\sqrt{2} \eta_{2}}{2}\right)
\end{aligned}
$$

for s-a.e. $y \in C_{0}[0, T]$ and a.e. $\eta_{j}(j=1,2,3,4) \in \mathbb{R}$. Also, both sides of the expression in equation (4.5) are given by the formula

$$
\begin{aligned}
& \int_{L^{2}[0, T]} \int_{L^{2}[0, T]} \exp \left\{\alpha \beta^{2} \tau\left\langle(v+u) h_{2}^{2} s_{2}, y\right\rangle\right. \\
& +\alpha^{2} \gamma^{2}\left(\left\|v h_{1}-B_{v h_{1}}\right\|_{2}^{2}+\left\|u h_{1}-B_{u h_{1}}\right\|_{2}^{2}\right) \\
& \left.+\frac{\sqrt{2} \alpha \gamma\left(\eta_{4}+\eta_{3}+\sqrt{2} \eta_{1}\right)}{2} B_{v h_{1}}+\frac{\sqrt{2} \alpha \gamma\left(\eta_{4}-\eta_{3}+\sqrt{2} \eta_{1}\right)}{2} B_{u h_{1}}\right\} d f(v) d g(u) .
\end{aligned}
$$

Proof. By using equation (4.1), it follows that

$$
\begin{aligned}
& T_{\gamma, \beta}^{h_{1}, h_{2}}\left(\left(T_{\gamma, \beta}^{h_{1}, h_{2}}(F \| X)\left(\cdot, \eta_{1}\right) *_{s_{1} s_{2}} T_{\gamma, \beta}^{h_{1}, h_{2}}(G \| X)\left(\cdot, \eta_{2}\right)\right)_{\rho, \tau}\left(\cdot, \eta_{3}\right) \| X\right)\left(y, \eta_{4}\right) \\
= & T_{\sqrt{2} \rho, \beta \tau}^{s_{1}, h_{2} s_{2}}\left(T_{\gamma, \beta}^{h_{1}, h_{2}}(F \| X)\left(\cdot, \eta_{1}\right) \| X\right)\left(y, \frac{\eta_{4}+\eta_{3}}{\sqrt{2}}\right) \\
& \cdot T_{\sqrt{2} \rho, \beta \tau}^{s_{1}, h_{2} s_{2}}\left(T_{\gamma, \beta}^{h_{1}, h_{2}}(G \| X)\left(\cdot, \eta_{1}\right) \| X\right)\left(y, \frac{\eta_{4}-\eta_{3}}{\sqrt{2}}\right) .
\end{aligned}
$$

Also, applying equation (3.9) to the last expression above, we obtain

$$
\begin{aligned}
& T_{\gamma, \beta}^{h_{1}, h_{2}}\left(\left(T_{\gamma, \beta}^{h_{1}, h_{2}}(F \| X)\left(\cdot, \eta_{1}\right) *_{s_{1} s_{2}} T_{\gamma, \beta}^{h_{1}, h_{2}}(G \| X)\left(\cdot, \eta_{2}\right)\right)_{\rho, \tau}\left(\cdot, \eta_{3}\right) \| X\right)\left(y, \eta_{4}\right) \\
= & T_{\sqrt{2} \gamma, \beta^{2} \tau}^{h_{1}, h_{2}^{2} s_{2}}(F \| X)\left(y, \frac{\eta_{4}+\eta_{3}+\sqrt{2} \eta_{1}}{2}\right) T_{\sqrt{2} \gamma, \beta^{2} \tau}^{h_{1}, h_{2}^{2} s_{2}}(G \| X)\left(y, \frac{\eta_{4}-\eta_{3}+\sqrt{2} \eta_{2}}{2}\right) .
\end{aligned}
$$

This proves the desired result.

Remark 4.4. Let $A \equiv\left\{w \in C_{0}[0, T]: w(t)=\int_{0}^{t} u(s) d s\right.$ for some $\left.u \in L^{2}[0, T]\right\}$. Note that for all $u, v \in L^{2}[0, T]$, we have that $\left|(u, v)_{2}\right| \leq\|u\|_{2}\|v\|_{2}$. In addition, for $w \in A$ and $v \in L^{2}[0, T]$, the PWZ integral $\langle v, w\rangle$ exists and is given by the formula

$$
\langle v, w\rangle=\int_{0}^{T} v(s) d w(s)=\int_{0}^{T} v(s) u(s) d s
$$

and so $|\langle v, w\rangle| \leq\|v\|_{2}\|u\|_{2}$. 
The following observation below will be very useful in the development of our theorems. Let $w \in A$ and let $F$ be an element of $S_{\alpha}$ whose associated measure $f \in \mathcal{M}\left(L^{2}[0, T]\right)$ satisfies the inequality

$$
\int_{L^{2}[0, T]}|\alpha|\|v\|_{2}|d f(v)|<\infty .
$$

Then $\delta F$ is an element of $S_{\alpha}$. Hence in this paper, we always assume that the associated measure $f \in \mathcal{M}\left(L^{2}[0, T]\right)$ of $F$ satisfies the condition (4.6).

In our next theorem, we establish that the conditional transform involving the first variation equals the first variation of the conditional transform with respect to the Gaussian process.

Theorem 4.5. Let $F$ and $X$ be as in Theorem 4.1. Assume that $F$ satisfies the hypothesis of Remark 4.4. Let $h, s, l, m$ and $h_{j}(j=1,2,3,4)$ satisfy the following conditions:

$$
\begin{aligned}
& \text { (1) } h_{3}(t)=h(t) h_{1}(t), \\
& \text { (2) } l(t) h_{4}(t)=h(t) h_{2}(t), \\
& \text { (3) } m(t) h_{4}(t)=s(t) \text { on }[0, T] .
\end{aligned}
$$

Then for all $(\gamma, \beta) \in E_{\alpha}$,

$$
T_{\gamma, \beta}^{h_{1}, h_{2}}\left(\delta F\left(Z_{h}(\cdot, \cdot) \mid Z_{s}(z, \cdot)\right) \| X\right)(y, \eta)=\frac{1}{\beta} \delta T_{\gamma, \beta}^{h_{3}, h_{4}}(F \| X)\left(Z_{l}(y, \cdot) \mid Z_{m}(z, \cdot), \eta\right)
$$

for s-a.e. $y \in C_{0}[0, T]$ and a.e. $\eta \in \mathbb{R}$. Also, both sides of the expression in equation (4.7) are given by the formula

$\int_{L^{2}[0, T]} \alpha\langle v s, z\rangle \exp \left\{\alpha \beta\left\langle v h h_{2}, y\right\rangle+\frac{\alpha^{2} \gamma^{2}}{2}\left\|v h h_{1}-B_{v h h_{1}}\right\|_{2}^{2}+\alpha \gamma \eta B_{v h h_{1}}\right\} d f(v)$.

Furthermore equation (4.7) is an element of $S_{\alpha \beta}$ with associated measure $\phi_{4}^{\eta}$ defined by

$$
\phi_{4}^{\eta}(E)=\int_{E} \alpha\langle v s, z\rangle \exp \left\{\frac{\alpha^{2} \gamma^{2}}{2}\left\|v h h_{1}-B_{v h h_{1}}\right\|_{2}^{2}+\alpha \gamma \eta B_{v h h_{1}}\right\} d f(v)
$$

for $E \in \mathcal{B}\left(L^{2}[0, T]\right)$.

Proof. First, using equations (2.5) and (2.9), we obtain that

$$
\begin{aligned}
& T_{\gamma, \beta}^{h_{1}, h_{2}}\left(\delta F\left(Z_{h}(\cdot, \cdot) \mid Z_{s}(z, \cdot)\right) \| X\right)(y, \eta) \\
= & \int_{C_{0}[0, T]} \delta F\left(Z_{h}\left(\gamma Z_{h_{1}}\left(x(\cdot)-\frac{\dot{T}}{T} x(T)+\frac{\dot{T}}{T} \eta\right)+\beta Z_{h_{2}}(y, \cdot), \cdot\right) \mid Z_{s}(z, \cdot)\right) d m(x) \\
= & \int_{C_{0}[0, T]} \frac{\partial}{\partial k}\left[\int _ { L ^ { 2 } [ 0 , T ] } \operatorname { e x p } \left\{\alpha \beta\left\langle v h h_{2}, y\right\rangle+\alpha \gamma\left\langle v h h_{1}-B_{v h_{1}}, x\right\rangle\right.\right.
\end{aligned}
$$




$$
\begin{gathered}
\left.\left.+\alpha \gamma \eta B_{v h h_{1}}+\alpha k\langle v s, z\rangle\right\} d f(v)\right]\left.\right|_{k=0} d m(x) \\
=\int_{L^{2}[0, T]} \alpha\langle v s, z\rangle \exp \left\{\alpha \beta\left\langle v h h_{2}, y\right\rangle+\alpha \gamma \eta B_{v h h_{1}}\right\} \\
\cdot\left[\int_{C_{0}[0, T]} \exp \left\{\alpha \gamma\left\langle v h h_{1}-B_{v h_{1}}, x\right\rangle\right\} d m(x)\right] d f(v) \\
=\int_{L^{2}[0, T]} \alpha\langle v s, z\rangle \exp \left\{\alpha \beta\left\langle v h h_{2}, y\right\rangle+\frac{\alpha^{2} \gamma^{2}}{2}\left\|v h h_{1}-B_{v h h_{1}}\right\|_{2}^{2}+\alpha \gamma \eta B_{v h h_{1}}\right\} d f(v) .
\end{gathered}
$$

Next, using equations (2.5) and (3.3), it follows that

$$
\begin{aligned}
& \delta T_{\gamma, \beta}^{h_{3}, h_{4}}(F \| X)\left(Z_{l}(y, \cdot) \mid Z_{m}(z, \cdot), \eta\right) \\
= & \left.\frac{\partial}{\partial k}\left[T_{\gamma, \beta}^{h_{3}, h_{4}}(F \| X)\left(Z_{l}(y, \cdot)+k Z_{m}(z, \cdot), \eta\right)\right]\right|_{k=0} \\
= & \frac{\partial}{\partial k}\left[\int _ { L ^ { 2 } [ 0 , T ] } \operatorname { e x p } \left\{\alpha \beta\left\langle v l h_{4}, y\right\rangle+\frac{\alpha^{2} \gamma^{2}}{2}\left\|v h_{3}-B_{v h_{3}}\right\|_{2}^{2}\right.\right. \\
& \left.\left.\quad+\alpha \gamma \eta B_{v h_{3}}+\alpha \beta k\left\langle v h_{4} m, z\right\rangle\right\} d f(v)\right]\left.\right|_{k=0} \\
= & \int_{L^{2}[0, T]} \alpha \beta\left\langle v h_{4} m, z\right\rangle \exp \left\{\alpha \beta\left\langle v l h_{4}, y\right\rangle+\frac{\alpha^{2} \gamma^{2}}{2}\left\|v h_{3}-B_{v h_{3}}\right\|_{2}^{2}+\alpha \gamma \eta B_{v h_{3}}\right\} d f(v) .
\end{aligned}
$$

Thus, equation (4.7) follows from equations (4.8) and (4.4).

Remark 4.6. The main result in [5, Theorem 4.2] follows immediately from Theorem 4.5 above by choosing $h_{1}=h_{2}=1$.

The following theorem follows immediately from Theorems 3.5 and 4.5.

Theorem 4.7. Let $F$ and $X$ be as in Theorem 4.5. Let $h, s, l, m$ and $h_{j}(j=$ $1,2,3,4,5,6)$ satisfy the following conditions:

(1) $h_{5}(t)=h(t) h_{3}(t)$,

(2) $l(t) h_{6}(t)=h(t) h_{2}(t) h_{4}(t)$

(3) $m(t) h_{6}(t)=s(t)$ on $[0, T]$.

Then for all $\left(\gamma_{2}, \beta_{2}\right) \in E_{\alpha}$ and $\left(\gamma_{2}, \beta_{1} \beta_{2}\right) \in E_{\alpha}$,

$$
\begin{aligned}
& T_{\gamma_{1}, \beta_{1}}^{h_{1}, h_{2}}\left(T_{\gamma_{2}, \beta_{2}}^{h_{3}, h_{4}}\left(\delta F\left(Z_{h}(\cdot, \cdot) \mid Z_{s}(z, \cdot)\right) \| X\right)\left(\cdot, \eta_{1}\right) \| X\right)\left(y, \eta_{2}\right) \\
= & \frac{1}{\beta_{1} \beta_{2}} \delta T_{\sqrt{2} \gamma_{2}, \beta_{1} \beta_{2}}^{h_{5}, h_{6}}(F \| X)\left(Z_{l}(y, \cdot) \mid Z_{m}(z, \cdot), \frac{\eta_{2}+\eta_{1}}{\sqrt{2}}\right)
\end{aligned}
$$

for s-a.e. $y \in C_{0}[0, T]$ and a.e. $\eta_{1}, \eta_{2} \in \mathbb{R}$. Also, both sides of the expression in equation (4.10) are given by the formula

$$
\int_{L^{2}[0, T]} \alpha\langle v s, z\rangle \exp \left\{\alpha \beta_{1} \beta_{2}\left\langle v h h_{2} h_{4}, y\right\rangle+\alpha^{2} \gamma_{2}^{2}\left\|v h h_{3}-B_{v h h_{3}}\right\|_{2}^{2}\right.
$$


CONDITIONAL TRANSFORM WITH RESPECT TO THE GAUSSIAN PROCESS 1575

$$
\left.+\alpha \gamma_{2}\left(\eta_{2}+\eta_{1}\right) B_{v h h_{3}}\right\} d f(v) .
$$

\section{Relationships between three concepts}

In Section 4, we obtained relationships between exactly two of the three concepts of conditional transform, conditional convolution product and first variation of functionals on $S_{\alpha}$. In this section, we establish all possible relationships between all three of these concepts.

In this section, to simplify the expressions, we will only state the formulas without conditions for existences.

Formula 5.1. Let $F$ and $X$ be as in Theorem 4.5. Let $h, s, l, m$ and $h_{j}$ $(j=1,2,3,4)$ satisfy the following conditions:

(1) $\tau \gamma=\rho$,
(2) $h_{1}(t) s_{2}(t)=l(t) h_{3}(t)=s_{1}(t)$,
(3) $h(t) h_{2}(t) s_{2}(t)=l(t) h_{4}(t)$
(4) $m(t)=h_{2}(t) s_{2}(t) s(t)$ on $[0, T]$.

Then

$$
\begin{aligned}
& \delta T_{\gamma, \beta}^{h_{1}, h_{2}}\left(\left(\left(F *_{s_{1} s_{2}} G\right)_{\rho, \tau} \| X\right)\left(\cdot, \eta_{1}\right) \| X\right)\left(Z_{h}(y, \cdot) \mid Z_{s}(z, \cdot), \eta_{2}\right) \\
= & T_{\sqrt{2} \rho, \tau \beta}^{s_{1}, h_{2} s_{2}}(F \| X)\left(Z_{h}(y, \cdot), \frac{\eta_{2}+\eta_{1}}{\sqrt{2}}\right) \tau \beta T_{\sqrt{2} \rho, \tau \beta}^{h_{3}, h_{4}}\left(\delta G\left(Z_{l}(\cdot, \cdot) \mid Z_{m}(z, \cdot)\right) \| X\right)\left(y, \frac{\eta_{2}-\eta_{1}}{\sqrt{2}}\right) \\
+ & \tau \beta T_{\sqrt{2} \rho, \tau \beta}^{h_{3}, h_{4}}\left(\delta F\left(Z_{l}(\cdot, \cdot) \mid Z_{m}(z, \cdot)\right) \| X\right)\left(y, \frac{\eta_{2}+\eta_{1}}{\sqrt{2}}\right) T_{\sqrt{2} \rho, \tau \beta}^{s_{1}, h_{2} s_{2}}(G \| X)\left(Z_{h}(y, \cdot), \frac{\eta_{2}-\eta_{1}}{\sqrt{2}}\right) .
\end{aligned}
$$

Formula 5.2. Let $F$ and $X$ be as in Theorem 4.5. Let $h, s, l, m$ and $h_{j}$ $(j=1,2,3,4)$ satisfy the following conditions:
(1) $\tau \gamma=\rho$,
(2) $h_{1}(t) s_{2}(t)=s_{1}(t)$,
(3) $h_{3}(t)=h(t) s_{1}(t)$
(4) $l(t) h_{4}(t)=h(t) h_{2}(t) s_{2}(t)$,
(5) $m(t) h_{4}(t)=s(t)$ on $[0, T]$.

Then

$$
\begin{aligned}
& T_{\gamma, \beta}^{h_{1}, h_{2}}\left(\left(\left(\delta F\left(Z_{h}(\cdot, \cdot) \mid Z_{s}(z, \cdot)\right) *_{s_{1} s_{2}} \delta G\left(Z_{h}(\cdot, \cdot) \mid Z_{s}(z, \cdot)\right)\right)_{\rho, \tau} \| X\right)\left(\cdot, \eta_{1}\right) \| X\right)\left(y, \eta_{2}\right) \\
= & \frac{1}{\beta^{2}} \delta T_{\sqrt{2} \rho, \tau \beta}^{h_{3}, h_{4}}(F \| X)\left(Z_{l}(y, \cdot) \mid Z_{m}(z, \cdot), \frac{\eta_{2}+\eta_{1}}{\sqrt{2}}\right) \delta T_{\sqrt{2} \rho, \tau \beta}^{h_{3}, h_{4}}(G \| X)\left(Z_{l}(y, \cdot) \mid Z_{m}(z, \cdot), \frac{\eta_{2}-\eta_{1}}{\sqrt{2}}\right) .
\end{aligned}
$$

Formula 5.3. Let $F$ and $X$ be as in Theorem 4.5. Let $h, s, l, m$ and $h_{j}$ $(j=1,2,3,4)$ satisfy the following conditions:
(1) $\tau \gamma=\rho$,
(2) $h_{3}(t) s_{2}(t)=s_{1}(t)$
(3) $h_{3}(t)=h(t) h_{1}(t)$,
(4) $l(t) h_{4}(t)=h(t) h_{2}(t)$
(5) $m(t) h_{4}(t)=s(t)$ on $[0, T]$. 
Then

$$
\begin{aligned}
& T_{\gamma, \beta}^{h_{1}, h_{2}}\left(\delta\left(\left(\left(F *_{s_{1} s_{2}} G\right)_{\rho, \tau} \| X\right)\left(\cdot, \eta_{1}\right)\right)\left(Z_{h}(\cdot, \cdot) \mid Z_{s}(z, \cdot)\right) \| X\right)\left(y, \eta_{2}\right) \\
= & T_{\sqrt{2} \rho, \gamma \beta}^{s_{1}, h_{2} s_{2}}(F \| X)\left(Z_{l}(y, \cdot), \frac{\eta_{2}+\eta_{1}}{\sqrt{2}}\right) \frac{1}{\beta} \delta T_{\sqrt{2} \rho, \gamma \beta}^{s_{1}, h_{2} s_{2}}(G \| X)\left(Z_{l}(y, \cdot) \mid Z_{m}(z, \cdot), \frac{\eta_{2}-\eta_{1}}{\sqrt{2}}\right) \\
& +\frac{1}{\beta} \delta T_{\sqrt{2} \rho, \gamma \beta}^{s_{1}, h_{2} s_{2}}(F \| X)\left(Z_{l}(y, \cdot) \mid Z_{m}(z, \cdot), \frac{\eta_{2}+\eta_{1}}{\sqrt{2}}\right) T_{\sqrt{2} \rho, \gamma \beta}^{s_{1}, h_{2} s_{2}}(G \| X)\left(Z_{l}(y, \cdot), \frac{\eta_{2}-\eta_{1}}{\sqrt{2}}\right) .
\end{aligned}
$$

Acknowledgments. The authors thank the referees for their helpful suggestions which led to the present version of this paper.

\section{References}

[1] R. H. Cameron, Some examples of Fourier-Wiener transforms of analytic functionals, Duke Math. J. 12 (1945), 485-488.

[2] R. H. Cameron and W. T. Martin, Fourier-Wiener transforms of functionals belonging to $L_{2}$ over the space C, Duke Math. J. 14 (1947), 99-107.

[3] S. J. Chang, H. S. Chung, and D. Skoug, Some basic relationships among transforms, convolution products, first variations and inverse transforms, Cent. Eur. J. Math. 11 (2013), no. 3, 538-551.

[4] J. G. Choi and S. J. Chang, A rotation on Wiener space with applications, ISRN Appl. Math. 2012 (2012), Art. ID 578174, 13 pages.

[5] H. S. Chung, J. G. Choi, and S. J. Chang, Conditional integral transforms with related topics on function space, Filomat 26 (2012), no. 6, 1151-1162.

[6] D. M. Chung, C. Park, and D. Skoug, Generalized Feynman integrals via conditional Feynman integrals, Michigan Math. J. 40 (1993), no. 2, 337-391.

[7] H. S. Chung, D. Skoug, and S. J. Chang, Relationships involving transform and convolutions via the translation theorem, Stoch. Anal. Appl. 32 (2014), no. 2, 348-363.

[8] H. S. Chung and V. K. Tuan, Generalized integral transforms and convolution products on function space, Integral Transforms Spec. Funct. 22 (2011), no. 8, 573-586.

[9] D. L. Cohn, Measure Theory, Birkhäuser-Verlag, Boston, 1980.

[10] G. W. Johnson and D. L. Skoug, Scale-invariant measurability in Wiener space, Pacific J. Math. 83 (1979), no. 1, 157-176.

[11] B. J. Kim, Conditional integral transforms, conditional convolution products and first variations for some conditioning functions, Far East J. Math. Sci. 19 (2005), no. 3, $245-258$.

[12] B. S. Kim, B. J. Kim, and D. Skoug, Conditional integral transforms, conditional convolution products and first variations, Panamer. Amer. Math. J. 14 (2004), no. 3, 27-47.

[13] B. S. Kim and D. Skoug, Integral transforms of functionals in $L_{2}\left(C_{0}[0, T]\right)$, Rocky Mountain J. Math. 33 (2003), no. 4, 1379-1393.

[14] I. Y. Lee, H. S. Chung, and S. J. Chang, Relationships among the transform with respect to the Gaussian process, the $\diamond$-product and the first variation of functionals on function space, to submitted for publications.

[15] C. Park and D. Skoug, A simple formula for conditional Wiener integrals with applications, Pacific J. Math. 135 (1988), no. 2, 381-394.

[16] W. Rudin, Real and Complex Analysis, McGraw-Hill, New York, 1966.

Hyun Soo Chung

DePARTMEnT OF MATHEMATiCs

DANKOOK UNIVERSITY

Cheonan 330-714, Korea

E-mail address: hschung@dankook.ac.kr 
CONDITIONAL TRANSFORM WITH RESPECT TO THE GAUSSIAN PROCESS 1577

IL YONG LEE

Department of Mathematics

DANKOOK UNIVERSITY

Cheonan 330-714, Korea

E-mail address: iylee@dankook.ac.kr

Seung Jun Chang

Department of Mathematics

DANKOOK UNIVERSITY

CheOnAn 330-714, Korea

E-mail address: sejchang@dankook.ac.kr 